\title{
ON APPROACH OF ESTIMATION TIME SCALES OF RELAXATION OF CONCENTRATION OF CHARGE CARRIERS IN HIGH-DOPED SEMI- CONDUCTOR
}

\author{
E.L. Pankratov ${ }^{1}$, E.A. Bulaeva ${ }^{1,2}$ \\ ${ }^{1}$ Nizhny Novgorod State University, 23 Gagarin avenue, \\ Nizhny Novgorod, 603950, Russia \\ ${ }^{2}$ Nizhny Novgorod State University of Architecture and Civil Engineering, \\ 65 Il'insky street, Nizhny Novgorod, 603950, Russia
}

\begin{abstract}
In this paper we generalized recently introduced approach for estimation of time scales of mass transport. The approach have been illustrated by estimation of time scales of relaxation of concentrations of charge carriers in high-doped semiconductor. Diffusion coefficients and mobility of charge carriers and electric field strength in semiconductor could be arbitrary functions of coordinate.
\end{abstract}

\section{KEYWORDS}

Time scales; approach of estimation; arbitrary spatial dependences of diffusion coefficient and potential profiles

\section{INTRODUCTION}

To date mathematical theory of mass transport has been elaborated in details [1-5]. However several problems attracted an interest. Some of them are: estimation of diffusion coefficient [6,7], diffusion length $[7,8]$, charge carrier mobility $[9,10]$. Some of important characteristics of nonstationary charge carrier transport are temporal characteristics. The above characteristics attracted an interest due to necessity of analysis of performance of semiconductor devices. Traditional way to determine time scales of charge carrier transport required determination non-stationary solution of appropriate mass transport equation. If parameters of semiconductor (charge carrier diffusion coefficients and mobilities et al) and strength of electric field in semiconductor material are independent on coordinate, mass transport equation could be easily solved [11,12]. In the common case of dependence of the above parameters and the electric field on coordinate exact solution of mass transport equation is unknown. Framework this paper we generalized recently introduced approach to estimate time characteristics of mass transport.

To illustrate the approach we consider one-dimension transport of charge carriers in a semiconductor material. Diffusion coefficients of charge carriers in the material (diffusion coefficient for electrons $D_{n}(x)$ and diffusion coefficient for holes $\left.D_{p}(x)\right)$ are arbitrary functions of coordinate $x$, $0 \leq x \leq L$. Distribution of electrical potential $\varphi(x)$ is also arbitrary function of coordinate. We assume, that we consider high-doped material. In this case we can neglect by non-primary charge carriers in comparison with primary one. We also assume, that at initial moment of time $t=0$ a distribution of charge carriers is presented in the considered material. With time these distribu- 
tions relaxating to their stationary distributions $n(x, \infty)$ и $p(x, \infty)$. Main aim of the present paper is estimation of relaxation time of stationary distributions of charge carriers concentrations.

\section{METHOd Of SOLUTiON}

Charge carriers concentrations framework drift-diffusion approximation have usually complex time dependences. In this situation it is necessary to approximate these time dependences by a function with simple time dependence. One of the simplest approximations could be written as: $\psi(x, t, \Theta)=a_{0}(x)+a_{.1}(x)[1(t)-1(t-\Theta(x))]$. Let us assume dependence of parameters $a_{0}(x)$, $a_{1}(x)$ and $\Theta(x)$ on coordinate $x$, but do not write the argument in future. We determine relaxation time of distributions of charge carriers concentrations as interval between moments of initial states of the considered concentrations and moment of step-wise changing of the function $\psi(x, t, \Theta)$. We determine values of parameters $a_{0}, a_{1}$ and $\Theta$ of approximation function by minimization of the following mean-squared error

$$
U=\int_{0}^{t_{N}}[C(x, t)-\psi(x, t, \Theta)]^{2} d t
$$

Here $t_{N}$ is the continuance of relaxation process, $C(x, t)$ is the concentration of any type of charge carriers. We consider spatio-temporal distributions of concentrations of charge carriers as a solution of the second Fick's law

$$
\begin{aligned}
& \frac{\partial n(x, t)}{\partial t}=\frac{1}{q} \frac{\partial \vec{j}_{n}(x, t)}{\partial x}, \\
& \frac{\partial p(x, t)}{\partial t}=\frac{1}{q} \frac{\partial \vec{j}_{p}(x, t)}{\partial x} .
\end{aligned}
$$

Here $\vec{j}_{n}(x, t)$ is the current density of electrons, $\vec{j}_{p}(x, t)$ is the current density of holes, $q$ is the elementary charge. Current densities include into itself two components: diffusion $\vec{j}_{\text {diff }}(x, t)$ and drift $\vec{j}_{d r}(x, t)$ components. These components could be written as

$$
\begin{gathered}
\vec{j}_{n d i f f}(x, t)=q D_{n}(x) \frac{\partial n(x, t)}{\partial x}, \vec{j}_{p d i f f}(x, t)=-q D_{p}(x) \frac{\partial p(x, t)}{\partial x} \\
\vec{j}_{n d r}(x, t)=-q n(x, t) \mu_{n}(x) \frac{d \varphi(x)}{d x}, \vec{j}_{p d r}(x, t)=-q n(x, t) \mu_{p}(x) \frac{d \varphi(x)}{d x} .
\end{gathered}
$$

Here $\mu_{n}(x)$ and $\mu_{p}(x)$ are the electron and hole mobilities, $D_{n}(x)$ and $D_{p}(x)$ are the diffusion coefficients of electron and hole. The second Fick's laws (2) and (3) with account the above approximations of current densities could be written as

$$
\frac{\partial n(x, t)}{\partial t}=\frac{\partial}{\partial x}\left[D_{n}(x) \frac{\partial n(x, t)}{\partial x}\right]-\frac{\partial}{\partial x}\left[\mu_{n}(x) n(x, t) \frac{d \varphi(x)}{d x}\right],
$$


International Journal of Information Technology, Modeling and Computing (IJITMC) Vol. 4, No.3, August 2016

$$
\frac{\partial p(x, t)}{\partial t}=\frac{\partial}{\partial x}\left[D_{p}(x) \frac{\partial p(x, t)}{\partial x}\right]+\frac{\partial}{\partial x}\left[\mu_{p}(x) p(x, t) \frac{d \varphi(x)}{d x}\right] .
$$

Boundary and initial conditions for the Eqs. (4) and (5) could be written as: $n(x, 0)=f_{n}(x)$, $p(x, 0)=f_{p}(x), \vec{j}_{n}(0, t)=\vec{j}_{n}(L, t)=\vec{j}_{p}(0, t)=\vec{j}_{p}(L, t)=0$. The Eqs. (4) and (5) are equal to each other after replacement $\varphi(x)$ on $-\varphi(x)$. It should be noted, that determination relaxation time for one type of charge carriers gives a possibility to determine the relaxation time for another type of charge carriers.

To determine relaxation time of distribution of concentration of charge carriers we determine optimal values of parameters of approximated function $\psi(x, t, \Theta)$ by minimization mean-squared error (1), i.e by using the following conditions

$$
\frac{\partial U}{\partial a_{0}}=0, \frac{\partial U}{\partial a_{1}}=0, \frac{\partial U}{\partial \Theta}=0 .
$$

The conditions (6) gives a possibility to obtain the following equations for calculation optimal value of parameters $a_{0}, a_{1}$ and $\Theta$

$$
\begin{gathered}
\int_{0}^{t_{N}} p(x, t) d t=a_{0} t_{N}+a_{1} \Theta, \\
\int_{0}^{\Theta} p(x, t) d t=\left(a_{0}+a_{1}\right) \Theta, \\
p(x, \Theta)=a_{0}+a_{1} / 2 .
\end{gathered}
$$

One can obtain analogous relations for concentration of electrons.

We obtain nonlinear equations for estimation of relaxation time. Solution of the equations do not attracted any serous problems. Increasing of continuance time $t_{N}$ leads to some changing of parameters $a_{0}, a_{1}$ and shifting of moment $\Theta$ of step-wise changing of approximation function.

To obtain analytical relations for relaxation time they are attracted an interest asymptotically optimal criteria. Framework the criteria continuance of relaxation time is unlimited, i.e. $t_{N} \rightarrow \infty$.

The criteria needs in additional condition on parameter $a_{1}: a_{1}=p(x, 0)-p(x, \infty)$. One shall determine moment of step-wise changing of approximation function $\Theta$ only. In this situation the required relaxation time could be calculated by solution of linear equation, i.e. we obtain linear criterion for estimation relaxation time. To obtain analytical solution of system of equations (7), (7a) and (7b) we consider them in the asymptotical case $t_{N} \rightarrow \infty$. To obtain the solution one shall to take into account, that in the limiting case $a_{0}$ became equal to $p(x, \infty)$. In the same limiting case Eq.(7) transforms to the following form

$$
\Theta_{p}(x)=\frac{\int_{0}^{\infty}[p(x, \infty)-p(x, t)] d t}{[p(x, \infty)-p(x, 0)]} .
$$


In this situation we obtain recently introduced asymptotically optimal approximation of temporal dependence of concentration of charge carriers by rectangle with equal square. One can obtain from Eq. (7b) another asymptotically optimal criterion to estimate time scales

$$
p(x, \Theta)=[p(x, 0)-p(x, \infty)] / 2 \text {. }
$$

However the criterion gives smaller quantity of analytical relations for relaxation time $\Theta$ in comparison with rectangle with equal square. It should be noted, that asymptotically optimal criteria could be used only for monotonous dependences of concentration on time. If these dependences are not monotonous, one shall use solve Eqs. (7)-(7b) in common case. In this situation to obtain analytical relations for relaxation time we will consider monotonous dependences of concentration on time only and will use criterion (8). To calculate relaxation times we use the following approach [13-15].

Let us consider Laplace transformation of concentration and current density of holes

$$
\begin{aligned}
& Y_{p}(x, s)=\int_{0}^{\infty} p(x, t) e^{-s t} d t, \\
& G_{p}(x, s)=\int_{0}^{\infty} j_{p}(x, t) e^{-s t} d t .
\end{aligned}
$$

Analogous relations could be written for concentration and current density of electrons.

Using the Laplace transformation relation (8) and Eqs. (4) and (5) could be written as

$$
\begin{gathered}
\frac{\partial}{\partial x}\left[D_{p}(x) \frac{\partial Y_{p}(x, s)}{\partial x}\right]+\frac{\partial}{\partial x}\left[\mu_{p}(x) Y_{p}(x, s) \frac{d \varphi(x)}{d x}\right]-s Y_{p}(x, s)=-f_{p}(x), \\
\Theta_{p}(x)=\lim _{s \rightarrow 0} \frac{p(x, \infty)-s Y_{p}(x, s)}{s[p(x, \infty)-p(x, 0)]} .
\end{gathered}
$$

To calculate relaxation time (12) it is not necessary to know concentration $Y_{p}(x, s)$ and current density $G_{p}(x, s)$ for all values of Laplace parameter $s$. It is necessary to know the above functions for asymptotical values of the parameter $s \rightarrow 0$. Let us consider the following power series

$$
s Y_{p}(x, s)=Z_{0}^{p}(x)+s Z_{1}^{p}(x)+s^{2} Z_{2}^{p}(x)+\ldots, s G_{p}(x, s)=H_{0}^{p}(x)+s H_{1}^{p}(x)+s^{2} H_{2}^{p}(x)+\ldots
$$

Substitution the relation (13) into the relation (12) leads to the following result

$$
\Theta_{p}(x)=\lim _{s \rightarrow 0} \frac{p(x, \infty)-Z_{0}^{p}(x)-s Z_{1}^{p}(x)-s^{2} Z_{2}^{p}(x)-\ldots}{s[p(x, \infty)-p(x, 0)]} .
$$

Limiting theorems of Laplace transformation [16] gives a possibility to obtain the following results

$$
\lim _{s \rightarrow 0} s Y_{p}(x, s)=p(x, \infty)=Z_{0}^{p}(x), \lim _{s \rightarrow 0} s G_{p}(x, s)=G_{p}(x, \infty)=H_{0}^{p}(x) .
$$


In this situation stationary distributions of charge carrier concentration and current density are equal to $Z_{0}^{p}(x)$ and $H_{0}^{p}(x)=0$. With account relations (15) the relaxation time (14) could be written as

$$
\Theta_{p}(x)=\frac{Z_{1}^{p}(x)}{p(x, 0)-p(x, \infty)} .
$$

To determine relaxation time one shall determine functions $Z_{0}^{p}(x)$ and $Z_{1}^{p}(x)$. Substitution of the series (13) into Eq.(11) gives a possibility to obtain equations for the functions $Z_{k}^{p}(x)$

$$
\left\{\begin{array}{l}
\frac{\partial}{\partial x}\left[D_{p}(x) \frac{\partial Z_{0}^{p}(x)}{\partial x}\right]+\frac{\partial}{\partial x}\left[\mu_{p}(x) Z_{0}^{p}(x) \frac{d \varphi(x)}{d x}\right]=0 \\
\frac{\partial}{\partial x}\left[D_{p}(x) \frac{\partial Z_{1}^{p}(x)}{\partial x}\right]+\frac{\partial}{\partial x}\left[\mu_{p}(x) Z_{1}^{p}(x) \frac{d \varphi(x)}{d x}\right]=Z_{0}^{p}(x)-f_{p}(x) . \\
\frac{\partial}{\partial x}\left[D_{p}(x) \frac{\partial Z_{k}^{p}(x)}{\partial x}\right]+\frac{\partial}{\partial x}\left[\mu_{p}(x) Z_{k}^{p}(x) \frac{d \varphi(x)}{d x}\right]=Z_{k-1}^{p}(x), k \geq 2
\end{array}\right.
$$

Correlation between functions $Z_{k}^{p}(x)$ and $H_{k}^{p}(x)$ could be written as

$$
-\frac{H_{k}^{p}(x)}{D_{p}(x)}=\frac{d Z_{k}^{p}(x)}{d x}+\frac{\mu_{p}(x) Z_{k}^{p}(x)}{D_{p}(x)} \frac{d \varphi(x)}{d x} .
$$

The first integral of the first equation of the system (17) could be written as

$$
D_{p}(x) \frac{d Z_{0}^{p}(x)}{d x}+\mu_{p}(x) Z_{0}^{p}(x) \frac{d \varphi(x)}{d x}=c^{p} .
$$

The integration constant $c^{p}$ is equal to zero due to boundary conditions. The second integral of the first equation of the system (17) takes the form

$$
Z_{0}^{p}(x)=c_{1}^{p} e^{-\beta_{p}(x)} .
$$

Here $\beta_{p}(x)=\int_{0}^{x} \frac{\mu_{p}(v)}{D_{p}(v)} \frac{d \varphi(v)}{d v} d v$. Boundary conditions give a possibility to determine the integration constant $c_{1}^{p}$. After the determination one can obtain stationary distribution of concentration with account of normalization in the following form

$$
Z_{0}^{p}(x)=\frac{e^{-\beta_{p}(x)}}{\rho_{p}(L)} .
$$

Here $\rho_{p}(L)=\int_{0}^{L} e^{-\beta_{p}(v)} d v$. Now we determine solution of the second equation of system (17). The first integral could be written as 


$$
D_{p}(x)\left[\frac{\mu_{p}(x)}{D_{p}(x)} \frac{d \varphi}{d x} Z_{1}^{p}+\frac{d Z_{1}^{p}}{d x}\right]=P_{p}(x)-\int_{0}^{x} f_{p}(v) d v+c_{2}^{p}=-H_{1}^{p}(x) .
$$

Here $P_{p}(L)=\rho_{p}(x) / \rho_{p}(L)$. Boundary conditions gives zero values of integration constant $c_{2}^{p}$. The second integral of the second equation of the system (17) takes the form

$$
Z_{1}^{p}(x)=\left[\int_{0}^{x} \frac{e^{\beta_{p}(y)}}{D_{p}(y)} P_{p}(y) d y-\int_{0}^{x} e^{\beta_{p}(y)} \int_{0}^{y} f_{p}(v) d v \frac{d y}{D_{p}(y)}+c_{3}^{p}\right] e^{-\beta_{p}(x)} .
$$

We determine integration constant $c_{3}^{p}$ by using boundary conditions and relation (18)

$$
c_{3}^{p}=\int_{0}^{L} e^{-\beta_{p}(x)} \int_{0}^{x} e^{\beta_{p}(y)} \int_{0}^{y} f_{p}(v) d v \frac{d y d x}{\rho_{p}(L) D_{p}(y)}-\int_{0}^{L} e^{-\beta_{p}(x)} \int_{0}^{x} e^{\beta_{p}(y)} \frac{P_{p}(y) d y d x}{\rho_{p}(L) D_{p}(y)} .
$$

The relation could be simplify to the following form

$$
c_{3}^{p}=\int_{0}^{L} \frac{e^{\beta_{p}(y)}}{D_{p}(y)}\left[1-P_{p}(y)\right] \int_{0}^{y} f_{p}(v) d v d y-\int_{0}^{L} \frac{e^{\beta_{p}(y)}}{D_{p}(y)} P_{p}(y)\left[P_{p}(y)-1\right] d y .
$$

Now we have a possibility to obtain the function $Z_{1}^{p}(x)$ in the final form

$$
\begin{aligned}
Z_{1}^{p}(x)=e^{-\beta_{p}(x)}\left\{\int_{0}^{x} \frac{e^{\beta_{p}(y)}}{D_{P}(y)}\right. & {\left.\left[P_{P}(y)-\int_{0}^{y} f_{P}(v) d v\right] d y+\int_{0}^{L} \frac{e^{\beta_{p}(y)}}{D_{P}(y)}\left[1-P_{P}(y)\right] \int_{0}^{y} f_{P}(y) d v d y\right\}- } \\
& -e^{-\beta_{p}(x)}\left\{\int_{0}^{L} e^{\beta_{p}(y)} \frac{P_{p}(y)}{D_{p}(y)}\left[P_{p}(y)-1\right] d y\right\} .
\end{aligned}
$$

After calculation functions $Z_{0}^{p}(x)$ and $Z_{1}^{p}(x)$ we have a possibility to determine the relaxation time (16)

$$
\begin{aligned}
\Theta_{p}(x)=\frac{1}{\rho_{p}(L) f_{p}(x) e^{\beta_{p}(x)}-1}\left\{\int_{0}^{x} \frac{e^{\beta_{p}(y)}}{D_{p}(y)}\left[P_{p}(y)-\frac{1}{\rho_{p}(L)} \int_{0}^{y} f_{p}(v) d v\right] d y+\right. \\
\left.+\int_{0}^{L} \frac{e^{\beta_{p}(y)}}{D_{p}(y)} \int_{0}^{y} f_{p}(v) d v\left[\rho_{p}(L)-\rho_{p}(y)\right] d y-\int_{0}^{L} e^{\beta_{p}(y)} \frac{\rho_{p}(y)}{D_{p}(y)}\left[P_{p}(y)-1\right] d y\right\} .
\end{aligned}
$$

Replacement $\varphi(x)$ on $-\varphi(x)$ in the relation (24) gives a possibility to obtain relaxation time for electrons. If electric field in the considered semiconductor sample is absent, drift terms in Eqs. (4) and (5) will be equal to zero. The relaxation time (24) will be also independent on electric potential $\varphi(x)$.

Example 1 Let us consider initial distribution of concentration of charge carriers in the following form: $f(x)=\delta\left(x-x_{0}\right)$. The initial distribution will be consider for all future examples. In this case relation (24) could be written as 
International Journal of Information Technology, Modeling and Computing (IJITMC) Vol. 4, No.3, August 2016

$$
\begin{aligned}
& \Theta_{p}\left(x \geq x_{0}\right)=\int_{0}^{L} \frac{e^{\beta_{p}(y)}}{D_{p}(y)}\left[P_{p}(y) \int_{y}^{L} e^{-\beta_{p}(v)} d v\right] d y-\int_{x}^{L} \frac{e^{\beta_{p}(y)}}{D_{p}(y)} \int_{y}^{L} e^{-\beta_{p}(v)} d v d y-\int_{0}^{x_{0}} \frac{e^{\beta_{p}(y)}}{D_{p}(y)} \rho_{p}(y) d y, \\
& \Theta_{p}\left(x \leq x_{0}\right)=\int_{0}^{L} \frac{e^{\beta_{p}(y)}}{D_{p}(y)}\left[P_{p}(y) \int_{y}^{L} e^{-\beta_{p}(v)} d v\right] d y-\int_{x_{0}}^{L} \frac{e^{\beta_{p}(y)}}{D_{p}(y)} \int_{y}^{L} e^{-\beta_{p}(v)} d v d y-\int_{0}^{x} \frac{e^{\beta_{p}(y)}}{D_{p}(y)} \rho_{p}(y) d y .
\end{aligned}
$$

Maximal relaxation time for the initial distribution could be obtain for $x_{0}=0$ and $x=L$. In this situation one can obtain the following relation for relaxation time

$$
\Theta_{p \max }=\int_{0}^{L} \frac{e^{\beta_{p}(y)}}{D_{p}(y)} P_{p}(y) \int_{y}^{L} e^{-\beta_{p}(v)} d v d y .
$$

If distance between points $x_{0}$ and $x$ decreases, the relaxation time (27) is also decreases.

Example 2 Let us consider the following constant parameters $\mu_{n}(x), \mu_{p}(x), D_{n}(x), D_{p}(x)$, $E(x)=-d \varphi / d x$. In this situation $\mu_{n}(x)=\mu_{0 n}, \quad \mu_{p}(x)=\mu_{0 p}, \quad D_{n}(x)=D_{0 n}, \quad D_{p}(x)=D_{0 p}$, $E(x)=E_{0}, \varphi(x)=b-x E_{0}$. Now we consider only maximal relaxation time because calculation in more common case is bulky. In this situation one can obtain the following result

$$
\begin{gathered}
\Theta_{n \max }=\frac{\mu_{n} E_{0} L\left[1+\exp \left(-\frac{\mu_{n} E_{0} L}{D_{0 n}}\right)\right]-2 D_{0 n}\left[1-\exp \left(-\frac{\mu_{n} E_{0} L}{D_{0 n}}\right)\right]}{\left(\mu_{n} E_{0}\right)^{2}\left[1-\exp \left(-\frac{\mu_{n} E_{0} L}{D_{0 n}}\right)\right]}, \\
\Theta_{p \max }=\frac{\mu_{p} E_{0} L\left[1+\exp \left(\frac{\mu_{p} E_{0} L}{D_{0 p}}\right)\right]-2 D_{0 p}\left[\exp \left(\frac{\mu_{p} E_{0} L}{D_{0 p}}\right)-1\right]}{\left(\mu_{p} E_{0}\right)^{2}\left[\exp \left(\frac{\mu_{p} E_{0} L}{D_{0 p}}\right)-1\right]} .
\end{gathered}
$$

It is attracted an interest asymptotic behavior of relaxation times (28)-(29). At small and large dimensions of the considered semiconductor sample the relaxation time is linear on $L$ : $\Theta_{p \max }=L / \mu_{p} E_{0}$. Analogous asymptotic behavior of relations (28)-(29) are the same at large and small values of diffusion coefficients. At the limiting case of small electric field Eqs. (4) and (5) will have only diffusion terms and maximal relaxation time will be equal to $\Theta_{p \max }=L^{2} / 6 D_{0}$.

This result coincides with result, obtained in [13] for zero electric field strength. At the large value of electric field strength: $\Theta_{p \max }=L / \mu_{p} E_{0}$. However at large value of $E_{0}$ dependence of relaxation time on $E_{0}$ will be compensated by nonlinear dependence of charge mobility on field $E_{0}$ : $\mu_{p}=\mu_{0 p} E_{c r} / E$ (the dependence could be used for $E \geq 4 E_{c r}$ ), where $E_{c r}$ is the critical strength of electric field. At $E=E_{c r}$ drift velocity of charge carriers $v_{d r}=\mu E$ became approximately equal to heat velocity $v_{h}=\sqrt{2 k T / m}$ (see, for example, [17]). Asymptotical behavior of relations (28)(29) at large values of mobilities is analogous to asymptotical behavior at large and small electric field. 
Example 3 Let us consider distribution of electric potential the same as at Boltzmann equilibrium, i.e. $\varphi=a x_{1}\left[\exp \left(-x / x_{1}\right)-1\right]-b x+c$ for $x \leq L / 2 ; \varphi=a x_{1}\left[\exp \left(-x / x_{1}\right)-1\right]-\left[\exp \left(-(x-L) / x_{1}\right)-\right.$ $\left.-\exp \left(-L / x_{1}\right)\right] a x_{1}-b x+c$ for $x \geq L / 2$. Electric field strength, which corresponds to the potential, could be written as $E=a \exp \left(-x / x_{1}\right)+b$ for $x \leq L / 2 ; E=a \exp \left(-L / x_{1}\right)+b$ for $x \geq L / 2$.

Calculation of the relaxation time leads to bulky result. In this situation we will not consider the result.

Temporal characteristics of charge carriers transport in semiconductor samples in the case of Boltzmann equilibrium electric potential is essentially smaller in comparison with temporal characteristics for linear distribution of electric potential.

\section{Conclusions}

In this paper we generalized recently introduced approach for estimation of time scales of mass transport. The approach have been illustrated by estimation of time scales of relaxation of concentrations of charge carriers in high-doped semiconductor. Diffusion coefficients and mobility of charge carriers and electric field strength in semiconductor could be any functions of coordinate.

\section{ACKNOWLEDGEMENTS}

This work is supported by the agreement of August 27, 2013 № 02.B.49.21.0003 between The Ministry of education and science of the Russian Federation and Lobachevsky State University of Nizhni Novgorod, educational fellowship for scientific research of Government of Russian, educational fellowship for scientific research of Government of Nizhny Novgorod region of Russia and educational fellowship for scientific research of Nizhny Novgorod State University of Architecture and Civil Engineering.

\section{REFERENCES}

[1] A.I. Raichenko. Mathematical theory of diffusion in applications (Kiev, Naukova Dumka, 1981).

[2] J. Crank. The mathematics of diffusion (Oxford university press, 1975).

[3] A.B. Grebene. Bipolar and MOS analogous integrated circuit design (New York: John Wyley and Sons, 1983).

[4] A.F. Corballo Sanchez, G. Gonzalez de la Cruz, Yu.G. Gurevich, G.N. Logvinov. Transient heat transport by carriers and phonons in semiconductors. Physical review B. Vol. 59 (16). P. 10630 (1999).

[5] E.L. Pankratov. Dopant diffusion dynamics and optimal diffusion time as influenced by diffusioncoefficient nonuniformity. Russian Microelectronics. Vol. 36 (1). P. 33-39 (2007).

[6] R. Ditchfield, D. Llera-Rodriguez, E.G. Seebauer. Semiconductor surface diffusion: Nonthermal effects of photon illumination. Phys. Rev. B. Vol. 61 (20). P.13710 (2000).

[7] T. Laine, K. Saarinen, P. Hautojarvi. Low-temperature positron diffusion in GaAs. Phys. Rev. B. Vol. 62 (12). P. 8058 (2000).

[8] W. P. Gillin, D.J. Dunstan. Strain and interdiffusion in semiconductor heterostructures. Phys. Rev. B. Vol. 50 (11). P. 7495 (1994).

[9] Ch. Pokhrel, N. Shakya, S. Purtee, B. Ellman, A.N. Semyonov, R.J. Twieg. Effect of mobile ions on charge mobility measurements. J. Appl. Phys. Vol. 101. P. 103706 (2007).

[10] F. Schindler, J. Geilker, W. Kwapil, W. Warta, M. C. Schubert. Hall mobility in multicrystalline silicon. J. Appl. Phys. Vol. 110. P. 043722 (2011).

[11] A.N. Tikhonov, A.A. Samarsky. The mathematical physics equations (Moscow, Science, 1972).

[12] Y.Y. Shan, P. Asoka-Kumar, K.G. Lynn, S. Fung, C.D. Beling. Field effect on positron diffusion in semi-insulating GaAs. Phys. Rev. B. Vol. 54. P. 1982 (1996). 
International Journal of Information Technology, Modeling and Computing (IJITMC) Vol. 4, No.3, August 2016

[13] A.N. Malakhov. Relaxation time of substance concentration in a medium with an arbitrary spacevarying diffusion coefficient. Radiophysics and Quantum Electronics. Vol. 40 (7). P. 591-598 (1997).

[14] A.N. Malakhov. Relaxation rates of the nonequilibrium concentration and flow of a substance in a medium with sink and arbitrary space-varying diffusion coefficient. Radiophysics and Quantum Electronics. Vol. 42 (6). P. 512-520 (1999).

[15] A.N. Malakhov, E.L. Pankratov. Relaxation time of material density in a medium with arbitrary space-varying diffusion coefficient and potential profile. Radiophysics and Quantum Electronics. Vol. 44 (6). P. 339-345 (2001).

[16] G. Korn, T. Korn. Mathematical Handbook for scientists and engineers. Definitions, theorems and formulas for reference and review. Second edition. McGraw-Hill Book Company. New York, 1968.

[17] I.P. Stepanenko. Basis of microelectronics (Moscow, Soviet Radio, 1980).

\section{AUTHORS}

Pankratov Evgeny Leonidovich was born at 1977. From 1985 to 1995 he was educated in a secondary school in Nizhny Novgorod. From 1995 to 2004 he was educated in Nizhny Novgorod State University: from 1995 to 1999 it was bachelor course in Radiophysics, from 1999 to 2001 it was master course in Radiophysics with specialization in Statistical Radiophysics, from 2001 to 2004 it was PhD course in Radiophysics. From 2004 to 2008 E.L. Pankratov was a leading technologist in Institute for Physics of Microstructures. From 2008 to 2012 E.L. Pankratov was a senior lecture/Associate Professor of Nizhny Novgorod State University of Architecture and Civil Engineering. 2012-2015 Full Doctor course in Radiophysical Department of Nizhny Novgorod State University. Since 2015 E.L. Pankratov is an Associate Professor of Nizhny Novgorod State University. He has 155 published papers in area of his researches.

Bulaeva Elena Alexeevna was born at 1991. From 1997 to 2007 she was educated in secondary school of village Kochunovo of Nizhny Novgorod region. From 2007 to 2009 she was educated in boarding school "Center for gifted children". From 2009 she is a student of Nizhny Novgorod State University of Architecture and Civil Engineering (spatiality "Assessment and management of real estate"). At the same time she is a student of courses "Translator in the field of professional communication" and "Design (interior art)" in the University. Since 2014 E.A. Bulaeva is in a PhD program in Radiophysical Department of Nizhny Novgorod State University. She has 103 published papers in area of her researches. 